\title{
The asymptotic core, nucleolus and Shapley value of smooth market games with symmetric large players
}

\section{Avishay Aiche, Anna Rubinchik \& Benyamin Shitovitz}

International Journal of Game Theory

ISSN 0020-7276

Int J Game Theory

DOI 10.1007/s00182-014-0422-1

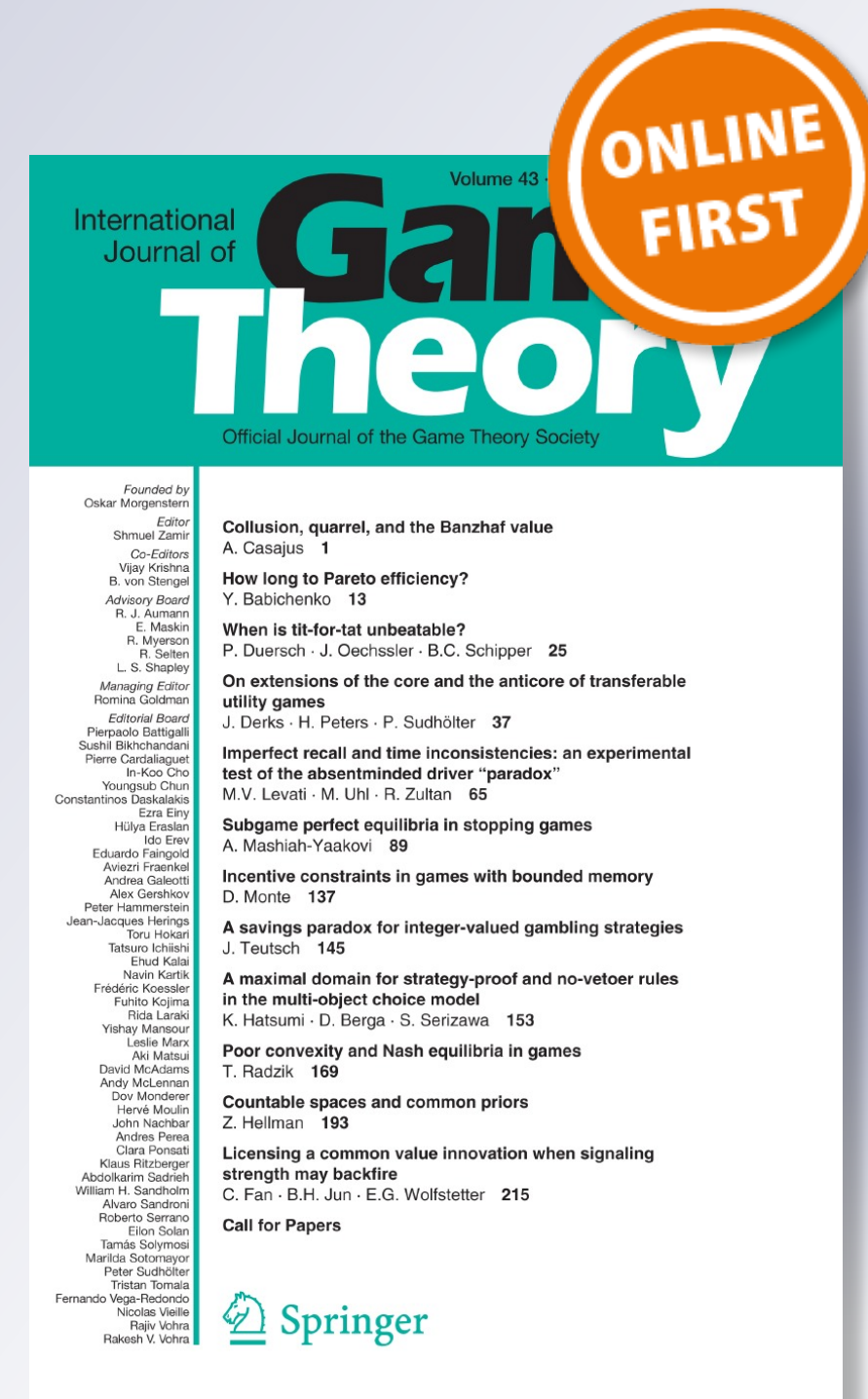

Springer 
Your article is protected by copyright and all rights are held exclusively by SpringerVerlag Berlin Heidelberg. This e-offprint is for personal use only and shall not be selfarchived in electronic repositories. If you wish to self-archive your article, please use the accepted manuscript version for posting on your own website. You may further deposit the accepted manuscript version in any repository, provided it is only made publicly available 12 months after official publication or later and provided acknowledgement is given to the original source of publication and a link is inserted to the published article on Springer's website. The link must be accompanied by the following text: "The final publication is available at link.springer.com". 


\title{
The asymptotic core, nucleolus and Shapley value of smooth market games with symmetric large players
}

\author{
Avishay Aiche - Anna Rubinchik • \\ Benyamin Shitovitz
}

Accepted: 27 March 2014

(C) Springer-Verlag Berlin Heidelberg 2014

\begin{abstract}
We examine the asymptotic nucleolus of a smooth and symmetric oligopoly with an atomless sector in a transferable utility (TU) market game. We provide sufficient conditions for the asymptotic core and the nucleolus to coincide with the unique TU competitive payoff distribution. This equivalence results from nucleolus of a finite TU market game belonging to its core, the core equivalence in a symmetric oligopoly with identical atoms and single-valuedness of the core in the limiting smooth game. In some cases (but not always), the asymptotic Shapley value is more favourable for the large traders than the nucleolus, in contrast to the monopoly case (Einy et al. in J Econ Theory 89(2):186-206, 1999), where the nucleolus allocation is larger than the Shapley value for the atom.
\end{abstract}

Keywords Mixed games · Oligopoly $\cdot$ Asymptotic nucleolus · Asymptotic Shapley value

JEL Classification $\quad$ C71 $\cdot$ D40 $\cdot$ D43

A. Aiche's contribution is part of his Ph.D. thesis being prepared at the University of Haifa under the supervision of Prof. B. Shitovitz.

A. Aiche · A. Rubinchik · B. Shitovitz ( $\square)$

Department of Economics, University of Haifa, Mount Carmel, 31905 Haifa, Israel

e-mail: binya@econ.haifa.ac.il

A. Aiche

e-mail: aaish@campus.haifa.ac.il

A. Rubinchik

e-mail: arubinchik@econ.haifa.ac.il

Published online: 16 April 2014 


\section{Introduction}

Consider a mixed differentiable game, proposed by Aumann (1964), with a few big (atomic) players and a continuum of small ones. Our task here is to compare their allocation under different solution concepts: competitive equilibrium versus core, nucleolus (defined for finite games in Schmeidler 1969) and Shapley value, and thus assess the "market power" of the large players embodied in each of the concepts. The comparison of core and competitive allocations in a general mixed game was done by Shitovitz (1973), and a comprehensive survey of the known results for the core of mixed games is in Gabszewicz and Shitovitz (1992).

We focus on transferable utility (TU) vector-measure games that include smooth production games. Our main result is that the asymptotic core (the limit of the cores of the finite games in the r-admissible sequence) of the smooth game with identical big players coincides with the unique transferable utility competitive equilibrium (t.u.c.e.) payoff.

As a consequence, the asymptotic (limiting) nucleolus is the t.u.c.e. payoff distribution as well. This is in contrast with the monopoly case, studied in Einy et al. (1999), where the nucleolus is strictly better for the monopoly than the t.u.c.e. Also, there the big player's asymptotic Shapley value is smaller than its nucleolus, which is no longer true in a game with two big players.

In the next section we describe the mixed smooth vector-measure (production) game, and verify that its core is non-empty and establish sufficient conditions for the core to be a singleton. In Sect. 3, we define an r-admissible sequence of finite games converging to the initial smooth game, generalising the classical "replica" sequences for mixed games and present our main results: characterisation of the limiting core and the nucleolus. In Sect. 4 we calculate the limiting Shapley value for a simple duopoly and compare it to core payoff for the big players in the original mixed game. The mixed game is represented as an Aumann-Shapley-Shubik game in Sect. 5, where we also provide the connection with the transferable utility competitive allocation and find that it is in the core of the original game.

\section{The game and its core}

Notation 2.1 Let $\mathscr{H}_{z}$ be the set of continuous, concave, super-additive functions $f: \mathbb{R}_{+}^{M} \rightarrow \mathbb{R}_{+}$, continuously differentiable in a neighbourhood of $z \in \mathbb{R}_{++}^{M}$, with $f(0)=0$.

\subsection{Definition of the game}

Let $T$ ( $=T_{0} \cup T_{1}$ ) be the set of players, $\Sigma$ be the set of all possible coalitions of the players and $\lambda$ be the "population measure" so that $(T, \Sigma, \lambda)$ is a measure space, where $\lambda$ is a $\sigma$-additive positive measure on $\Sigma$. The set of players is partitioned into $T_{1}$, the big players (the atoms of $\lambda$ ) and $T_{0}$, the small players, i.e., $\lambda=\lambda_{0}+\lambda_{1}$, where $\lambda_{0}$ is non-atomic (with support $T_{0}$ ), and $\lambda_{1}$ has finite support $T_{1}$. 
Let $\mu=\left(\mu_{1}, \ldots, \mu_{M}\right)$ be a vector of countably additive measures absolutely continuous with respect to $\lambda$, the vector represents distribution of resources. Assume $\mu(T) \in \mathbb{R}_{++}^{M}$. Let $V=f \circ \mu$ be the vector-measure game, with $f \in \mathscr{H}_{\mu(T)}$.

Remark 1 Concavity, super-additivity, non-negativity of $f$ and $f(0)=0$ imply homogeneity of degree one of $f$. Super-additivity and non-negativity of $f$ implies it is non-decreasing on $\mathbb{R}_{+}^{M}$. This implies, in particular, $\nabla f(\mu(T)) \geq 0$.

\subsection{Games with a partition structure}

For some applications we will further assume that $T$ is partitioned by a finite collection of subsets $\left\{A_{l}\right\}_{l \in L}: \cup_{l} A_{l}=T$, for any $l \neq l^{\prime}, A_{l} \cap A_{l^{\prime}}=\emptyset$. Let $\omega_{m}(t) \stackrel{\text { def }}{=} \frac{d \mu_{m}(t)}{d \lambda}$ be the Radon-Nikodym derivative on $T_{0}$ for all $m . \mu$ is constant on (the atoms) $T_{1} \cap A_{l}$ for any $l$.

The greatest common divisor $r$ of the numbers of big players in different elements of the partition, $\left\{T_{1} \cap A_{l}\right\}_{l \in L}$ is assumed to be at least 2 , hence monopoly case is excluded.

\subsection{The core}

Lemma $1 x^{*}(S) \stackrel{\text { def }}{=} p \mu(S)$ is in the core of $V$, where $p=\nabla f(\mu(T))$.

Proof Since $f$ is homogeneous of degree one by Remark 1 and is $C^{1}$ in the neighbourhood of $\mu(T)$, by Euler's theorem, $p \mu(T)=f(\mu(T))$ and by concavity of $f$ for all $z \in \mathbb{R}_{+}^{M}, f(z) \leq f(\mu(T))+p(z-\mu(T))=p z$. Thus for all $S \in \Sigma$, $x^{*}(S)=p \mu(S) \geq f(\mu(S))=V(S)$. The conclusion then follows by definition of the core.

Proposition 1 The core of a smooth vector-measure game where all big players have the same resources is the singleton, $\left\{x^{*}\right\}$.

Proof By Lemma $1 x^{*}$ is in the core. Assume now that $u^{*}$ is also in the core. By a standard argument used in Einy et al. (1999, pp.194-195), it is easy to see that the game $V$ is a continuous game at $T$ and $u^{*}(\cdot)$ is a non-negative, countably additive measure and absolutely continuous with respect to the population measure $\lambda$.

Next, we will show that $u^{*}(S)=x^{*}(S)=p \mu(S)$ for all $S \in \Sigma$ where $p=$ $(\nabla f)(\mu(T))$.

Step 1 The 'equal-treatment' property for the atoms: $\forall l \in L, a_{1}, a_{2} \in T_{1} \cap A_{l}$ implies $u^{*}\left(a_{1}\right)=u^{*}\left(a_{2}\right)$.

Assume by contradiction that $u^{*}\left(a_{1}\right)<u^{*}\left(a_{2}\right)$ for some $l^{\prime} \in L$ and $a_{1}, a_{2} \in T_{1} \cap A_{l^{\prime}}$. Denote by $\frac{1}{r}\left(T_{1} \cap A_{l}\right)$ the subset of $T_{1} \cap A_{l}$ who receive (according to $u^{*}$ ) at most as much as others within their partition element $A_{l}$ and possess $\frac{1}{r}$-th share of the resources: $\mu\left(\frac{1}{r}\left(T_{1} \cap A_{l}\right)\right)=\frac{1}{r} \mu\left(T_{1} \cap A_{l}\right)$. Then $u^{*}\left(\frac{1}{r}\left(T_{1} \cap A_{l}\right)\right) \leq \frac{1}{r} u^{*}\left(T_{1} \cap A_{l}\right)$ with strict inequality for $l=l^{\prime}$. Apply now Lyapunov's theorem for the vector measure 
$\left(u^{*}(\cdot), \mu(\cdot)\right)$ with respect to the non-atomic sector $T_{0} \subset T$ to obtain a subset $\frac{1}{r} T_{0} \subset T_{0}$ with $\left(u^{*}\left(\frac{1}{r} T_{0}\right), \mu\left(\frac{1}{r} T_{0}\right)\right)=\frac{1}{r}\left(u^{*}\left(T_{0}\right), \mu\left(T_{0}\right)\right)$. Let now $\frac{1}{r} T$ denote the disjoint union $\frac{1}{r} T_{0} \cup\left[\cup_{l \in L} \frac{1}{r}\left(T_{1} \cap A_{l}\right)\right]$. Then, by construction, $u^{*}\left(\frac{1}{r} T\right)<\frac{1}{r} u^{*}(T)$ and $\mu\left(\frac{1}{r} T\right)=$ $\frac{1}{r} \mu(T)$ but this contradicts the definition of the core, since $u^{*}\left(\frac{1}{r} T\right) \geq f\left(\mu\left(\frac{1}{r} T\right)\right)=$ $\frac{1}{r} f(\mu(T))=\frac{1}{r} u^{*}(T)$, where the first equality holds since $f \in \mathscr{H}_{\mu(T)}$ and the second equality is $u^{*}(T)=f(\mu(T))$.

Step $2 u^{*}(S)=p \mu(S)$ for all $S \subset T_{0}, S \in \Sigma$.

Apply Lyapunov's theorem for the vector measure $\left(u^{*}(\cdot), \mu(\cdot)\right)$ with respect to the two non-atomic sectors $S \subset T_{0}$ and $T_{0} \backslash S$. Thus, for each $0<\alpha<1$ there is $S_{\alpha} \subset S$ which has the monotonic property of inclusion with respect to $\alpha$ and $\frac{1}{r}\left(T_{0} \backslash S\right) \subset T_{0} \backslash S$ satisfying: $\left(u^{*}\left(S_{\alpha}\right), \mu\left(S_{\alpha}\right)\right)=\alpha\left(u^{*}(S), \mu(S)\right)$ and $\left(u^{*}\left(\frac{1}{r}\left(T_{0} \backslash S\right)\right), \mu\left(\frac{1}{r}\left(T_{0} \backslash S\right)\right)\right)=$ $\frac{1}{r}\left(u^{*}\left(T_{0} \backslash S\right), \mu\left(T_{0} \backslash S\right)\right)$.

So, take an arbitrary $S \subset T_{0}, S \in \Sigma$.

First, pick $0<\alpha<\frac{1}{2}$, small enough such that $f$ is still differentiable at $\frac{1}{r} \mu(T)+\alpha \mu(S)$, and let $\frac{1}{r} S=S_{\frac{1}{r}+\frac{1}{2}} \backslash S_{\frac{1}{2}}$ and $\frac{1}{r} T=\frac{1}{r} T_{1} \cup \frac{1}{r}\left(T_{0} \backslash S\right) \cup \frac{1}{r} S$. Then $\left(u^{*}\left(\frac{1}{r} T\right), \mu\left(\frac{1}{r} T\right)\right)=\frac{1}{r}\left(u^{*}(T), \mu(T)\right)$ and, since $S_{\alpha} \subset S_{\frac{1}{2}}$ and $\frac{1}{r} S \cap S_{\frac{1}{2}}=\varnothing$ by construction, $\frac{1}{r} T \cap S_{\alpha}=\varnothing$, hence $\frac{1}{r} u^{*}(T)+\alpha u^{*}(S)=u^{*}\left(\frac{1}{r} T \cup S_{\alpha}\right)$, which is $\geq f\left(\mu\left(\frac{1}{r} T\right)+\alpha \mu(S)\right)$, since $u^{*}$ is in the core. Therefore

$$
\alpha u^{*}(S) \geq f\left(\frac{1}{r} \mu(T)+\alpha \mu(S)\right)-f\left(\frac{1}{r} \mu(T)\right)
$$

which is $\geq(\nabla f)\left(\frac{1}{r} \mu(T)+\alpha \mu(S)\right) \alpha \mu(S)$ by concavity of $f$. Therefore

$$
u^{*}(S) \geq(\nabla f)\left(\frac{1}{r} \mu(T)+\alpha \mu(S)\right) \mu(S)
$$

Since $f \in \mathscr{H}_{\mu(T)}$, as $\alpha \rightarrow 0, u^{*}(S) \geq(\nabla f)\left(\frac{1}{r} \mu(T)\right) \mu(S)$, and since $f$ is homogeneous of degree one, $(\nabla f)\left(\frac{1}{r} \mu(T)\right)=(\nabla f)(\mu(T))$, so

$$
u^{*}(S) \geq p \mu(S)
$$

Now pick $0<\alpha<\frac{1}{r}$, small enough such that $f$ is still differentiable at $\frac{1}{r} \mu(T)-\alpha \mu(S)$, and let $\frac{1}{r} S=S_{\frac{1}{r}}$. Then $S_{\alpha} \subset S_{\frac{1}{r}}$, by the monotonic inclusion property. Again, let $\frac{1}{r} T=\frac{1}{r} T_{1} \cup \frac{1}{r}\left(T_{0} \backslash S\right) \cup \frac{1}{r} S$, then $\left(u^{*}\left(\frac{1}{r} T\right), \mu\left(\frac{1}{r} T\right)\right)=\frac{1}{r}\left(u^{*}(T), \mu(T)\right)$ and $\frac{1}{r} T \supset S_{\alpha}$. Therefore,

$$
\begin{aligned}
\frac{1}{r} u^{*}(T)-\alpha u^{*}(S) & =u^{*}\left(\frac{1}{r} T \backslash S_{\alpha}\right) \geq f\left(\mu\left(\frac{1}{r} T\right)-\alpha \mu(S)\right) \Longrightarrow \\
u^{*}(S) & \leq(\nabla f)\left(\frac{1}{r} \mu(T)-\alpha \mu(S)\right) \mu(S)
\end{aligned}
$$


Similarly, as $f \in \mathscr{H}_{\mu(T)}$, letting $\alpha \rightarrow 0$ we get

$$
u^{*}(S) \leq(\nabla f)\left(\frac{1}{r} \mu(T)\right) \mu(S)=p \mu(S)
$$

Since (1) and (2) hold for an arbitrary coalition $S \subset T_{0}$, we have $u^{*}(S)=x^{*}(S)$ for any such $S$, as required. It follows the same is true for any $S \subset T_{1}$, since all the big players have the same resources and therefore should be treated equally by step 1 . Hence $u^{*}=x^{*}$.

In the next section we will show that in a vector-measure game of proposition 1 the core of the mixed game coincides with the asymptotic core and with the asymptotic nucleolus.

\section{Asymptotic core and nucleolus}

\subsection{The admissible sequence of finite games}

Although the upper-hemicontinuity of the core has been studied in Trockel (1976), we focus only on the limit along a classical "replica" sequence of games: at any step the grand coalition $T$ can be partitioned into $r \geq 2$ identical coalitions (replicas), denoted $\frac{1}{r} T$, containing players from all elements of the partition (both big and small). However restrictive, this approach allows us to keep the proofs transparent, all the limiting solution concepts here easy to characterise and our results directly comparable to several previous important contributions.

Definition 1 Let $r \geq 2$ be, as defined above, the greatest common divisor $r$ of the numbers of big players in different elements of the partition, $\left\{T_{1} \cap A_{l}\right\}_{l \in L}$, with $L$ being a finite set. An $r$-admissible sequence of finite games for a given $\pi_{1}$ is defined by an increasing sequence, $\left(\pi_{n}\right)_{n=1}^{\infty}$, of finite subfields of $\Sigma$, with the corresponding set of all atoms $\Pi_{n}$ of $\pi_{n}$, such that

(1) $\pi_{n} \subset \pi_{n+1}$ for every $n=1,2 \ldots$;

(2) every subset of $T_{1}$ is in $\pi_{1}$;

(3) for every $l, T_{0} \cap A_{l} \in \pi_{1}$;

(4) for any $n$, and for any $l$ any two "small" players $i_{n}, j_{n} \in \Pi_{n}$, such that $i_{n}, j_{n} \subset$ $T_{0} \cap A_{l}$, have the same resources $\mu\left(i_{n}\right)=\mu\left(j_{n}\right)$;

(5) For every $n$ and any $l$ there is a natural number $k_{l n}$ such that $\mu\left(A_{l} \cap T_{0}\right)=$ $k_{\ln } r \mu\left(i_{n}\right)$

(6) $\cup_{n=1}^{\infty} \pi_{n}$ generates $\Sigma$.

Denote the restriction of the game $V$ to $\pi_{n}$ by $V_{n}$. Thus, $V_{n}(S) \equiv V\left(\cup_{i \in S} i\right)$ for any $S \subset \Pi_{n}$.

A measure $x$ is an $r$-asymptotic core measure of the vector-measure game $V$ if for any $S \in \Sigma$ and for any $r$-admissible sequence $\left(\pi_{n}\right)_{n=1}^{\infty}$ such that $S \in \pi_{1}$, there is a sequence of core measures $x_{n}$ of the finite games $V_{n}$, such that $x(S)=\lim _{n \rightarrow \infty} x_{n}(S)$. 
r-asymptotic nucleolus and r-asymptotic Shapley value are defined similarly (replacing 'core' in the paragraph above by 'nucleolus' and 'Shapley value' correspondingly).

Remark 2 (1) $\Pi_{n}$ can be thought of as the set of all players of the finite game, with the set of all its subsets (coalitions) being identified with $\pi_{n}$, as in Einy et al. (1999, p.191). All big players are in $\Pi_{n}$ for every $n$.

(2) Existence of an $r$-admissible sequence follows from Lyapunov-Richter theorem (cf. Mas-Collel 1985, L.1.3).

(3) In general, existence of an $r$-asymptotic solution imposes less restrictions than the corresponding classical asymptotic solution since in the latter case only independence with respect to $r$-admissible sequences is required. Hence any game that has an asymptotic core (nucleolus, Shapley value) has the $r$-asymptotic core (nucleolus, Shapley value) but the opposite is not necessarily true.

(4) If a vector-measure game has an asymptotic Shapley value (i.e., it belongs to ASY M P cf. Aumann and Shapley 1974; Neyman 1979, 2002; Neyman and Smorodinsky 2004), then this value is also its r-asymptotic value.

\section{2 r-Asymptotic core}

Theorem 1 Let $x_{n}$ be in the core of $V_{n}$. Then $\lim _{n \rightarrow \infty} x_{n}(S)=p \mu(S)\left(=x^{*}(S)\right)$ for every $S \subset T_{0}, S \in \pi_{1}$.

Proof Take $S \in \pi_{1}$, then it is a finite union of some disjoint coalitions $i \in \pi_{1}$ (or players in $\Pi_{1}$ ). If $S$ is also a subset of $T_{0}$, all such $i$ are subsets of $T_{0}$ too. Since the admissible sequence is increasing, $S \in \pi_{n}$ for any $n$.

By Lemma 1 the core of any (finite) game in the admissible sequence is non-empty. Further, since all the players of the same element $A_{l}$ within $T_{0}$ have the same resources (cf. def. 1.4), by step 1 of Proposition 1, all such players are treated equally in the core of any finite game $V_{n}$. The same holds for $T_{1}$.

Let $B_{n} \subset \Pi_{n}$ be the finite set of disjoint $i_{n} \subset T_{0}$ such that their union is $S$. For any game $V_{n}$ and any (small) players $i_{n}, i_{n}^{\prime} \in B_{n}$, with $i_{n} \notin \frac{1}{r} \Pi_{n}, i_{n}^{\prime} \in \frac{1}{r} \Pi_{n}$, since $x_{n}$ is a core allocation, ${ }^{1}$

$$
\begin{aligned}
& x_{n}\left(\frac{1}{r} \Pi_{n}\right)+x_{n}\left(\left\{i_{n}\right\}\right)=x_{n}\left(\frac{1}{r} \Pi_{n} \cup\left\{i_{n}\right\}\right) \geq V_{n}\left(\frac{1}{r} \Pi_{n} \cup\left\{i_{n}\right\}\right)=f\left(\frac{1}{r} \mu(T)+\mu\left(i_{n}\right)\right), \\
& x_{n}\left(\frac{1}{r} \Pi_{n}\right)-x_{n}\left(\left\{i_{n}^{\prime}\right\}\right)=x_{n}\left(\frac{1}{r} \Pi_{n} \backslash\left\{i_{n}^{\prime}\right\}\right) \geq V_{n}\left(\frac{1}{r} \Pi_{n} \backslash\left\{i_{n}^{\prime}\right\}\right)=f\left(\frac{1}{r} \mu(T)-\mu\left(i_{n}^{\prime}\right)\right)
\end{aligned}
$$

Since $x_{n}$ is additive (being a measure), by the equal treatment property of core allocations, $x_{n}\left(\frac{1}{r} \Pi_{n}\right)=\frac{1}{r} x_{n}\left(\Pi_{n}\right)=\frac{1}{r} V_{n}\left(\Pi_{n}\right)$ which equals $\frac{1}{r} f(\mu(T))$. Note that any coalition $\Pi_{n} \backslash \frac{1}{r} \Pi_{n}$ from the finite game $V_{n}$ contains players from all $\left(A_{l}\right)_{l \in L}$, so for any $i \in B_{n}, i \subset A_{l}$ there is $i_{n} \in \frac{1}{r} \Pi_{n}$ such that $i_{n} \subset A_{l}$ and $i_{n}^{\prime} \in \Pi_{n} \backslash \frac{1}{r} \Pi_{n}$ such that $i_{n}^{\prime} \subset A_{l}$. Therefore, $\mu\left(i_{n}\right)=\mu(i)=\mu\left(i_{n}^{\prime}\right)$ and by the equal treatment property of the core allocation $x_{n}, x_{n}\left(\left\{i_{n}\right\}\right)=x_{n}(\{i\})=x_{n}\left(\left\{i_{n}^{\prime}\right\}\right)$.

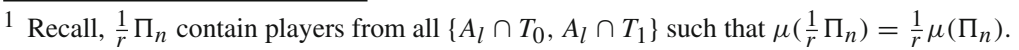


Combining with the inequalities above, we get

$f\left(\frac{1}{r} \mu(T)+\mu(i)\right)-f\left(\frac{1}{r} \mu(T)\right) \leq x_{n}(\{i\}) \leq f\left(\frac{1}{r} \mu(T)\right)-f\left(\frac{1}{r} \mu(T)-\mu(i)\right)$

By the homogeneity of $f, \nabla f(\mu(T))=\nabla f\left(\frac{1}{r} \mu(T)\right)$ and since $f$ is (continuously) differentiable in the neighbourhood of $\mu(T)$, it is so in an $\varepsilon$-neighbourhood of $\frac{1}{r} \mu(T)$. For any such $\varepsilon$ there is $N_{0}$ sufficiently large, such that for all $n>N_{0}$ one can assure $\|\mu(i)\|<\varepsilon$ for any $i \in B_{n}$, so for $n>N_{0}$, by concavity of $f$, the last inequality can be re-written as

$$
\nabla f\left(\frac{1}{r} \mu(T)+\mu(i)\right) \mu(i) \leq x_{n}(\{i\}) \leq \nabla f\left(\frac{1}{r} \mu(T)-\mu(i)\right) \mu(i)
$$

By continuity of $\nabla f$ in the $\varepsilon$-neighbourhood of $\frac{1}{r} \mu(T)$, there is $\bar{\delta} \in \mathbb{R}_{++}^{M}$ such that for any $0<\delta<\bar{\delta}$ there is $N_{1} \geq N_{0}$ such that for any $n>N_{1}$, and $i \in B_{n}$, $\nabla f\left(\frac{1}{r} \mu(T)+\mu(i)\right) \geq \nabla f(\mu(T))-\delta=p-\delta$. Similarly, there is $N_{2}>N_{0}$ such that $\forall n>N_{2}$, and $i \in B_{n}, \nabla f\left(\frac{1}{r} \mu(T)-\mu(i)\right) \leq p+\delta$. So, for all $n>\max \left\{N_{1}, N_{2}\right\}$ the inequality becomes $\forall i \in B_{n}$,

$$
(p-\delta) \mu(i) \leq x_{n}(\{i\}) \leq(p+\delta) \mu(i)
$$

Summing over all $i \in B_{n}$,

$$
(p-\delta) \mu(S) \leq x_{n}(S) \leq(p+\delta) \mu(S)
$$

Taking the limit as $\delta \rightarrow 0$, we get the result.

Corollary 1 Assume all the big players have the same resources. Then the $r$ asymptotic core is $\left\{x^{*}\right\}$.

Proof The "equal treatment" applies to big players, each of whom gets $\frac{p\left(\mu(T)-\mu\left(T_{0}\right)\right)}{\left|T_{1}\right|}$.

\section{3 r-Asymptotic nucleolus}

Corollary 2 Let $\mathcal{N}_{n}$ be the nucleolus of $n$-th game in the admissible sequence. Then $\lim _{n \rightarrow \infty} \mathcal{N}_{n}(S)=p \mu(S)$ for every $S \subset T_{0}, S \in \pi_{1}$.

Proof By Schmeidler (1969, thm. 4) nucleolus belongs the core of every finite game with non-empty core. The core of every game in the admissible sequence is non-empty by Lemma 1. The result follows from thm. 1 .

Remark 3 By Corollary 2 the non-atomic sector in our symmetric oligopoly obtains exactly twice as much as it gets in the monopolistic case, see Einy et al. (1999, theorem 3.1). 
Corollary 3 Assume all the big players have the same resources. Then the r-asymptotic nucleolus is $\left\{x^{*}\right\}$.

Proof Follows from cor. 1.

\section{Asymptotic shapley value for a simple duopoly game}

Recall, our objective is to study the limiting behaviour of the basic cooperative solution concepts. Here we focus on a simple set-up with all the small ("ocean") players being of the same type, as in Guesnerie (1977). For these games, as we just showed in Corollary 3, the asymptotic nucleolus is $\left\{x^{*}\right\}$. In case of monopoly (one atom), nucleolus allocation to the atom is higher than that of the Shapley value, see Einy et al. (1999), while in case of duopoly the comparison can go either way, as we show below.

Consider a simple-duopoly version of the smooth vector-measure game. There are two elements in the partition of players $(L=2)$. Let $\lambda_{0}$ be the Lebesgue measure on $T_{0}=[0,1] . A_{1}=T_{1}$ and $A_{2}=T_{0}$ and for all $t \in T_{0} \omega(t)=\omega_{0}$ and so their total endowment is $\omega_{0}$. Each of the two big players $\left(a_{1}, a_{2}\right)$ has endowment $\omega_{1}$, they are of equal size: $\lambda_{1}\left(a_{i}\right)=1$. Let $f: \mathbb{R}_{+}^{M} \rightarrow \mathbb{R}$ be concave, non-decreasing with $f(0)=0$ and continuous at zero and let $\mu(S)=\lambda_{0}([0,1] \cap S) \omega_{0}+\lambda_{1}\left(\left\{a_{1}, a_{2}\right\} \cap S\right) \omega_{1}$ such that $\omega_{0}+2 \omega_{1} \in \mathbb{R}_{++}^{M}$.

By Proposition 6 in Appendix B, the simple-duopoly game $V=f \circ \mu$ has an asymptotic Shapley value. By Remark 2(4), it follows that the game $V$ has a unique r-asymptotic Shapley value.

Now assume, in addition, that $\omega_{0} \in \partial \mathbb{R}_{+}^{M}$ and $f\left(\omega_{0}\right)=0$.

Proposition 2 The $r(=2)$-asymptotic Shapley value for the simple duopoly game allocates $\int_{0}^{1}\left[f\left(t \omega_{0}+\omega_{1}\right)+t\left(f\left(t \omega_{0}+2 \omega_{1}\right)-2 f\left(t \omega_{0}+\omega_{1}\right)\right)\right] d t$ to any big player.

Proof Any (finite) game in an $(r=2)$-admissible sequence should have $n$ small players ( $n$ even) and two big ones. Each of the small players holds $\frac{1}{n} \omega_{0}$. Since $f\left(t \omega_{0}\right)=$ 0 , the contribution of any big player to a coalition of $k$ small players is $h_{1}\left(\frac{k}{n}\right)$, where $h_{1}: t \mapsto f\left(t \omega_{0}+\omega_{1}\right)$; while the contribution to a coalition already containing a big player and $k$ small ones is $h_{2}\left(\frac{k}{n}\right), h_{2}: t \mapsto f\left(t \omega_{0}+2 \omega_{1}\right)-f\left(t \omega_{0}+\omega_{1}\right)$.

There are $(n+2)$ ! possible permutations of all players. For any position $k$ of the first big player there are $(n+2-k) n$ ! permutations where the second big player follows the first, while in the rest $((k-1) n$ !) the opposite is true. Therefore Shapley value for a big player $a_{i}$ is

$$
\begin{aligned}
{\left[\phi V_{n}\right]\left(\left\{a_{i}\right\}\right) } & =\frac{n !}{(n+2) !}\left[\sum_{k=2}^{n+2}(k-1) h_{2}\left(\frac{k-2}{n}\right)+\sum_{k=1}^{n+1}(n+2-k) h_{1}\left(\frac{k-1}{n}\right)\right] \\
& =\sum_{k=2}^{n+2} \frac{1}{n+1} \frac{k-1}{n+2} h_{2}\left(\frac{k-2}{n}\right)+\sum_{k=1}^{n+1} \frac{1}{n+1}\left(1-\frac{k}{n+2}\right) h_{1}\left(\frac{k-1}{n}\right)
\end{aligned}
$$

To establish the claim it is sufficient to show that the first summand converges to $\int_{0}^{1} t h_{2}(t) d t$ and the second one converges to $\int_{0}^{1}(1-t) h_{1}(t) d t$ as $n \rightarrow \infty$. 
We demonstrate the latter in detail, the proof of the former is analogous and is left to the reader. Let $g_{n}(t)=\left(1-\frac{n t+1}{n+2}\right) h_{1}(t)$, then the second term can be written as a Lebesgue integral $\int_{0}^{1} z_{n}(t) d \mu$ of a simple function $z_{n}$ returning a constant value $g_{n}\left(\frac{k-1}{n}\right)$ on $\left(\frac{k-1}{n}, \frac{k}{n}\right]$ for $k \in\{1, n+1\}$, by definition of Lebesgue integral, since $g_{n} \geq 0$ and

$$
\int_{0}^{1} z_{n}(t) d \mu=\sum_{k=1}^{n+1} \frac{1}{n+1} g_{n}\left(\frac{k-1}{n}\right) \leq h_{1}(1)<\infty \quad \forall n
$$

It is then left to show that $z_{n}$ converges uniformly on $[0,1]$ to the function $t \mapsto$ $(1-t) h_{1}(t)$. Indeed, by triangular inequality,

$$
\sup _{t}\left|(1-t) h_{1}(t)-z_{n}(t)\right| \leq \sup _{t}\left|g_{n}(t)-(1-t) h_{1}(t)\right|+\sup _{t}\left|z_{n}(t)-g_{n}(t)\right|
$$

The first term can be made arbitrarily small for large enough $n$ :

$$
\sup _{t}\left|\left(\frac{n t+1}{n+2}-t\right) h_{1}(t)\right| \leq \frac{2}{n+2} h_{1}(1)
$$

The same is true for the second term, since $f\left(\omega_{0}\right)=0$ and is monotonic, and so $h_{1}$ and $g_{n}$ on $[0,1]$ are of bounded variation. Thus, the uniform convergence.

Example 1 Let $f(x, y)=\sqrt{x y}$ and $\omega_{0}=(x, 0), \omega_{1}=(0, y)$ then the Shapley value to a big player is above his payoff in r-asymptotic nucleolus. Indeed, the latter is equal to $\mu\left(\left\{a_{1}\right\}\right) p=\omega_{1} \nabla f\left(\omega_{0}+2 \omega_{1}\right)$, and so here it is $\frac{1}{2 \sqrt{2}} \sqrt{x y}$, which is smaller than the Shapley value, $\left.\sqrt{x y}\left(\frac{2}{3}-(2-\sqrt{2}) \frac{2}{5}\right)\right)$, by Proposition 2 .

However, as in the monopoly case, the nucleolus payoff to the big player can be higher than the Shapley value:

Example 2 Let $f(x, y)=\left(\frac{1}{x}+\frac{1}{y}\right)^{-1}$ on $\mathbb{R}_{++}^{2}$ and 0 on the boundary. Let $\omega_{0}=$ $(8,0), \omega_{1}=(0,1)$. The $r$-asymptotic nucleolus payoff of a big player is, as before, $\omega_{1} \nabla f\left(\omega_{0}+2 \omega_{1}\right)$, and so is equal to $\frac{\partial f}{\partial y}(8,2)=0.64$. Again, by Proposition 2 , his Shapley value is:

$$
\begin{aligned}
& \int_{0}^{1}\left[\left(\frac{1}{8 t}+1\right)^{-1}+t\left(\left(\frac{1}{8 t}+1 / 2\right)^{-1}-2\left(\frac{1}{8 t}+1\right)^{-1}\right)\right] d t= \\
& {[-(5 \ln (8 x+1)) / 32+\ln (4 x+1) / 8+3 x / 4]_{0}^{1} \approx 0.607<0.64}
\end{aligned}
$$

\section{The Aumann-Shapley-Shubik game as a smooth vector-measure game}

We consider now a mixed version of the Shapley and Shubik (1969), Aumann and Shapley (1974), Einy et al. (1999) game and show that it can be represented as the 
smooth vector-measure game $V$ defined in Sect. 2.1. For a finite number of players, the notion of the "market game", an exchange economy with money with traders whose utility is concave and continuous in goods and additively linear in money, has been introduced by Shapley and Shubik (1969). Since here we require the "utilities" to be non-negative, a more natural interpretation, that of a production game is adopted.

Notation 5.1 Let a collection $\left(\int_{A} x_{m}(t) d \lambda(t)\right)_{m=1}^{M}$, with $A \subset T, x_{m}(t) \in \mathbb{R}_{+}$be denoted simply by $\int_{A} x(t) d \lambda(t)$ for $x(t) \in \mathbb{R}_{+}^{M}$.

Denote by $\mathscr{F}$ the set of all continuous functions $F: \mathbb{R}_{+}^{M} \rightarrow \mathbb{R}_{+}$that are concave, super-additive on $\mathbb{R}_{+}^{M}$ with $F(0)=0$ such that their partial derivatives $F^{m} \equiv \frac{\partial F}{\partial z_{m}}(z)$ exist for every $m$ at each point $z \in \mathbb{R}_{+}^{M}$ with $z_{m}>0$.

Let $\mathscr{F} 0$ contain all functions in $\mathscr{F}$ that are vanishing on the boundary of $\mathbb{R}_{+}^{M}$.

Remark 4 By Aumann and Shapley (1974, thm. 39.1) for any $z \in \mathbb{R}_{++}^{M}, \mathscr{F} \subset \mathscr{H}_{z}$.

Let us define a production game with $M$ commodities and initial distribution of endowments $\mu$.

Definition 2 A game $((T, \Sigma, \lambda), V)$ is a differentiable production game if there are $M$ positive countably additive measures $\left(\mu_{m}\right)_{m \in\{1, \ldots, M\}}$, absolutely continuous with respect to $\lambda$ with $\mu(T) \in \mathbb{R}_{++}^{M}$, and a collection $\left(F_{t}\right)_{t \in T}, F_{t} \in \mathscr{F}$, such that $V=\hat{V}$, where

$$
\hat{V}(S)=\sup _{\substack{x: T \rightarrow \mathbb{R}_{+}^{M} \\ \lambda \text {-integrable }}}\left\{\int_{S} F_{t}(x(t)) d \lambda(t): \int_{S} x(t) d \lambda(t) \leq \mu(S)\right\}
$$

Remark 5 If $f \in \mathscr{F}$, then the smooth market game $f \circ \mu$ is a production game, since one can set $F_{t}=f$ for all $t$. Indeed, by concavity and homogeneity of $f$, $\hat{V}(S) \leq \sup \left\{f\left(\int_{S} x(t) d \lambda(t)\right): \int_{S} x(t) d \lambda(t) \leq \mu(S)\right\}$ and by monotonicity of $f$, it is $\leq f(\mu(S))$. On the other hand, setting $x_{m}(t)=\frac{\mu(S)_{m}}{\lambda(S)}, t \in S$ for $\lambda(S)>0$ and $x_{m}(t)=0$ for $t \notin S$, yields $\hat{V}(S) \geq f(\mu(S))$, hence $\hat{V}=f \circ \mu$.

Assume now that there is a finite number of production functions $\left\{F_{t}\right\}_{t \in T}=\left\{F_{l}\right\}_{l \in L}$, $F_{l} \in \mathscr{F}$, and denote by $A_{l}$ the set of players $\left\{t \in T: F_{t}=F_{l}\right\}$.

Lemma 2 The production game $\hat{V}$ with a finite number of production functions is well defined, i.e., $\hat{V}(S)$ is finite for every $S$ and the supremum is attained.

Proof For any $l$ and any $S$ such that $\lambda\left(S \cap A_{l}\right)>0$ by concavity of $F_{l}$,

$$
\int_{S \cap A_{l}} F_{l}(x(t)) d \lambda(t) \leq \lambda\left(S \cap A_{l}\right) F_{l}\left(\frac{1}{\lambda\left(S \cap A_{l}\right)} \int_{S \cap A_{l}} x(t) d \lambda(t)\right)
$$

and since $\int_{S \cap A_{l}} x(t) d \lambda(t) \leq \mu(S)$, the right hand side is finite. Proposition 5 (with the set $\{1, \ldots, L\}$ replaced by $\left.\left\{l: \lambda\left(S \cap A_{l}\right)>0\right\}\right)$ then establishes that the 'supremum' in the definition of $\hat{V}(S)$ (Eq. (3)) can be replaced by 'maximum'. 
Remark 6 The conclusion might be false if the set of production functions is infinite. Indeed, let there be a countable set of production functions, set $M=1$ and $F_{l}(z) \equiv$ $\frac{l}{l+1} z$ then it is obvious that the supremum in definition 2 is not attained.

Using the notation of Sect. 2.2, $\mu(S)=\int_{S} \omega(t) d \lambda(t)$ for some integrable $\omega$ with values in $\mathbb{R}_{+}^{M}$. Recall, $\mu(T) \in \mathbb{R}_{++}^{M}$.

Also, there is full specialisation, i.e., $F_{l} \in \mathscr{F}_{0} \forall l$ and that each element $A_{l}$ has a corner endowment: ${ }^{2}$ for every $l \in\{1, \ldots, L\}$ there is an input $m_{l} \in\{1, \ldots, M\}$ such that $\omega(t)_{m_{l}}>0$ for $t$ in a subset of $A_{l}$ of positive measure, but none of the other types has that input: $\forall l^{\prime} \neq l \omega(t)_{m_{l}}=0$ for any $t \in A_{l^{\prime}}$. Thus, $\mu\left(A_{l}\right)_{m_{l}}>0$ and $\mu\left(A_{l^{\prime}}\right)_{m_{l}}=0$ for all $l^{\prime} \neq l$.

Proposition 3 The production game $\hat{V}$ with a finite number of production functions and full specialisation can be represented as a vector-measure game $V=f \circ \mu$, where $f(z) \stackrel{\text { def }}{=} \max _{\left(y_{l}\right)_{l}}\left\{\sum_{l} F_{l}\left(y_{l}\right): \sum_{l} y_{l} \leq z\right\}, f \in \mathscr{H}_{\mu(T)}$.

Proof First, it is sufficient to show that $f \in \mathscr{F}_{0}$. Indeed, by Remark $4 f \in \mathscr{F}_{0}$ implies $f \in \mathscr{H}_{z}$ for any $z \in \mathbb{R}_{++}^{M}$, hence $V=f \circ \mu$ is a smooth vector-measure game.

Consider a coalition $S$ such that $\lambda\left(S \cap A_{l}\right)>0$ for all $l \in L$. We will show now that there is $f \in \mathscr{F}$ such that $\hat{V}(S)=f(\mu(S))$ for all such coalitions $S$. By Lemma 2, for any $S$ with $\lambda\left(S \cap A_{l}\right)>0 \forall l$ the maximisation problem in the definition of $\hat{V}(S)$ reduces to the problem of optimal allocation of inputs across $L$ technologies, i.e., finding $\left(X_{l}\right)_{l=1}^{L} \in \mathbb{R}_{+}^{M L}$ (with $X_{l}=\int_{S \cap A_{l}} x(t) d \lambda(t)$ ) that maximises continuous function $\left(X_{l}\right)_{l} \mapsto \sum_{l} \lambda\left(S \cap A_{l}\right) F_{l}\left(X_{l}\right)$ on a compact support $\sum_{l} \lambda\left(S \cap A_{l}\right) X_{l} \leq \int_{S} \omega(t) d \lambda(t)$. By homogeneity of $F_{l}$, this problem is equivalent to

$$
\max _{\left(x_{l}\right)_{l}}\left\{\sum_{l} F_{l}\left(\lambda\left(S \cap A_{l}\right) X_{l}\right): \sum_{l} \lambda\left(S \cap A_{l}\right) X_{l} \leq z\right\}
$$

Define $f$ as in the statement of the proposition. Then, by construction, $\hat{V}(S)=$ $f(\mu(S))$ for $S: \lambda\left(S \cap A_{l}\right)>0 \forall l$. By Proposition 5 in the appendix, $f \in \mathscr{F}$. Obviously, if every $F_{l}$ vanishes on the boundary, so is $f$, hence, since $F_{l} \in \mathscr{F}_{0}$, so is $f$.

Now consider a coalition $S$ that lacks at least one element of the partition $A_{l}$, so that $\lambda\left(S \cap A_{l}\right)=0$ for some $l \in L$. Then by full specialisation assumptions, $\mu(S)$ has to be on the boundary of $\mathbb{R}_{+}^{M}$, and since $F_{l} \in \mathscr{F}_{0}$ for all $l \in L$ so is the maximum (over all allocations $x(t): \int_{S} x(t) d \lambda(t) \leq \mu(S)$ ) of their sum, hence $\hat{V}(S)=0$ in this case. But also with the same definition of $f$ as above, $f(\mu(S))=0$ for any such coalition. Hence, in this case, too $\hat{V}(S)=0=f(\mu(S))$.

So there is $f \in \mathscr{F}_{0}$ such that $\hat{V}(S)=f(\mu(S))$ for any $S \in \Sigma$, hence $\hat{V}$ is a smooth vector-measure game defined in Sect. 2.1.

\footnotetext{
2 Note it does not imply $L=M$ : with 3 commodities and $L=\{1,2\}, \omega_{1}=(1,1,0)$ and $\omega_{2}=(0,1,1)$ are corner endowments of a non-trivial game.
} 
Remark 7 Note it is not sufficient to assume that $F_{l}$ are in $\mathscr{F}$. Take, for example an economy with two goods and $L=\{1,2\}$ with endowments $(2,0),(0,2)$ and production functions $F_{1}(x, y)=x+2 y, F_{2}(x, y)=2 x+y$ (for each element correspondingly). The two big players (atoms) are of the first type and the small (non-atomic) players are of the second type. Clearly, $F_{1}, F_{2} \in \mathscr{F} \backslash \mathscr{F}_{0}$. Let $S_{0}$ be the coalition containing one big player and let $S_{\varepsilon}$ be the coalition containing a big player and a fraction $\varepsilon$ of the small players. Then by definition $\hat{V}\left(S_{0}\right)=2$ and $\hat{V}\left(S_{\varepsilon}\right)=4+4 \varepsilon$ and as $\varepsilon \rightarrow 0$ clearly, $\hat{V}\left(S_{\varepsilon}\right)$ is not converging to $\hat{V}\left(S_{0}\right)$. Hence, $\hat{V}$ can not be represented as $f \circ \mu$ with $f \in \mathscr{F}$ and $\mu$ countably additive measure, absolutely continuous with respect to $\lambda$. The reason for the discontinuity is that $F_{1}, F_{2}$ do not vanish on the boundaries.

Corollary 4 If the assumptions of Proposition 3 hold and if all the big players have the same resources, the core of the game $V$ and the core of $\hat{V}$ are both the singleton $\left\{x^{*}\right\}$.

Proof By Proposition 3 and 1.

Single-valuedness of the r-asymptotic core and nucleolus for symmetric oligopoly follows from Corollaries 1 and 3 respectively.

\subsection{Market game as a smooth vector measure game}

If we return to the more traditional interpretation, i.e., take $F_{t}$ as utilities of consumer $t$, we get an endowment economy, and so the "classical" market game. In this setting, as one would expect, the competitive equilibrium payoff distribution is in the core, by Corollary 5 below, which also implies that core equivalence holds if all the atoms have the same resources and $r \geq 2$.

Mainly to introduce the notation, we provide the definition below.

Definition 3 A transferable utility competitive equilibrium (t.u.c.e.) of the economy $\left(T, \Sigma, \lambda, F_{t}, \omega(t)\right)$ where $F_{t} \in \mathscr{F}$ is an allocation $z^{*}: T \rightarrow \mathbb{R}_{+}^{M}$ and a price $p^{*} \in$ $\mathbb{R}_{+}^{M}$ that satisfy (1) material balance: $\int_{T} z^{*}(t) d \lambda(t) \leq \mu(T)$; (2) zero net money supply: $p^{*}\left(\mu(T)-\int_{T} z^{*}(t) d \lambda(t)\right)=0$; (3) consumer optimisation: for $\lambda$-a.e. $t \in T$, $z^{*}(t)$ solves $\max _{z \in \mathbb{R}_{+}^{M}} U_{t}\left(z, p^{*} \omega(t)-p^{*} z\right)$, where $U_{t}(z, y): \mathbb{R}_{+}^{M} \times \mathbb{R} \rightarrow \mathbb{R}:$ $U_{t}(z, y) \stackrel{\text { def }}{=} F_{t}(z)+y$.

The t.u.c.e. payoff distribution is then $u^{*}(S) \stackrel{\text { def }}{=} \int_{S}\left[F_{t}\left(z^{*}(t)\right)+p^{*} \omega(t)-p^{*} z^{*}(t)\right]$ $d \lambda(t)$.

Lemma 3 If $\left(z^{*}, p^{*}\right)$ is a t.u.c.e. (of the economy with $\left.F_{t} \in \mathscr{F}\right)$ then $\max _{z \in R_{+}^{M}}\left[F_{t}(z)-\right.$ $\left.p^{*} z\right]=0$ and $F_{t}\left(z^{*}(t)\right)=p^{*} z^{*}(t)$ for $\lambda$-a.e. $t \in T$.

Proof Ignore $t$ in the negligible set of players for whom consumer optimisation fails to hold. For any other $t$

$$
\max _{z \in R_{+}^{M}}\left[F_{t}(z)-p^{*} z\right]=F_{t}\left(z^{*}(t)\right)-p^{*} z^{*}(t)
$$


Since $F_{t}(z)-p^{*} z$ is homogeneous of degree one in $z$, its maximum is either zero or $+\infty$. By material balance, $z^{*}(t)$ is finite for $\lambda$-a.e. $t$, hence for those $t$ the maximum is finite and thus is zero.

Proposition 4 Assume there is a finite number of production functions, with $F_{l} \in \mathscr{F}$ $\forall l$, then $u^{*}(S)=\nabla f(\mu(T)) \mu(S)$ for all $S \in \Sigma$, where $f$ is as defined in Proposition 3.

Remark 8 Note that we no longer assume full specialisation (in particular, $F_{l}$ are not necessarily vanishing on the boundaries), neither we assume that $F_{l}$ are strictly increasing.

Proof By Lemma 3, $u^{*}(S)=p^{*} \int_{S} \omega(t) d \lambda(t)=p^{*} \mu(S)$. It is then left to show that the equilibrium price $p^{*}$ equals $\nabla f(\mu(T))$.

First, we show that $p^{*} \leq \nabla f(\mu(T))$. By Proposition 5 in the appendix $F_{l} \in \mathscr{F} \Longrightarrow$ $f \in \mathscr{F}$, and so $\nabla f(\mu(T)) \geq 0$ and so, for $m$ such that $p_{m}^{*}=0$ the inequality holds.

If $p_{m}^{*}>0$, by definition of t.u.c.e. (1 and 2), $p_{m}^{*}\left[\mu_{m}(T)-\int_{T} z_{m}^{*}(t) d \lambda(t)\right]=0$ which yields $\int_{T} z_{m}^{*}(t) d \lambda(t)=\mu_{m}(T)>0$. Thus, for such $m$ there is at least one $l_{m} \in L$ with $\int_{A_{l_{m}}} z_{m}^{*}(t) d \lambda(t)>0$. Then, with $\overline{y_{l}} \stackrel{\text { def }}{=} \int_{A_{l}} z^{*}(t) d \lambda(t)$ for every $l \in L$, $\bar{y}_{l_{m}}^{m}>0$.

${ }^{m}$ For any $l$, integrating the identity in Lemma 3 over $A_{l}$ yields $p^{*} \bar{y}_{l}=$ $\int_{A_{l}} F_{l}\left(z^{*}(t)\right) d \lambda(t)$, on the other hand, by Lemma $3, F_{l}(x)-p^{*} x \leq 0$ for any $x \in \mathbb{R}_{+}^{M}$, and so $F_{l}\left(\bar{y}_{l}\right)=F_{l}\left(\int_{A_{l}} z^{*}(t) d \lambda(t)\right) \leq p^{*} \int_{A_{l}} z^{*}(t) d \lambda(t)=p^{*} \bar{y}_{l}$. By concavity, $F_{l}\left(\int_{A_{l}} z^{*}(t) d \lambda(t)\right) \geq \int_{A_{l}} F_{l}\left(z^{*}(t)\right) d \lambda(t)$, hence $F_{l}\left(\bar{y}_{l}\right)=p^{*} \bar{y}_{l}$. Thus, by Lemma $3, \bar{y}_{l}$ maximises $F_{l}(x)-p^{*} x$ over $x \in \mathbb{R}_{+}^{m}$.

Then, first, since $\bar{y}_{l_{m}}^{m}>0$ and $F_{l}$ is concave and differentiable there, $F_{l_{m}}^{m}\left(\bar{y}_{l_{m}}\right)=p_{m}^{*}$. Second, this implies $F_{l}\left(y_{l}\right)-p^{*} y_{l} \leq 0=F_{l}\left(\overline{y_{l}}\right)-p_{l}^{*} \overline{y_{l}}$ for $\left(y_{l}\right)_{l}$ that satisfy $\sum_{l \in L} y_{l} \leq \mu(T), y_{l} \in \mathbb{R}_{+}^{M}$. Thus, $F_{l}\left(\overline{y_{l}}\right) \geq F_{l}\left(y_{l}\right)+p^{*}\left(\overline{y_{l}}-y_{l}\right)$ and summing over $L$ we get $\sum_{l} F_{l}\left(\overline{y_{l}}\right) \geq \sum_{l} F_{l}\left(y_{l}\right)+p^{*} \sum_{l}\left(\overline{y_{l}}-y_{l}\right)$. But definition of t.u.c.e. (1 and 2), $p^{*} \sum_{l} \overline{y_{l}}=p^{*} \int_{T} z^{*}(t) d \lambda(t)=p^{*} \mu(T) \geq p^{*} \sum_{l} y_{l}$ for every $\left(y_{l}\right)_{l}$ that satisfy $\sum_{l} y_{l} \leq \mu(T)$. Therefore $\sum_{l} F_{l}\left(\overline{y_{l}}\right) \geq \sum_{l} F_{l}\left(y_{l}\right)$ for all such $\left(y_{l}\right)_{l}$, hence $\sum_{l} F_{l}\left(\bar{y}_{l}\right)=f(\mu(T))$. Apply now Proposition 5 for $z^{*}=\mu(T)$ and $\left(\bar{y}_{l}\right)_{l \in L}$ satisfying $\sum_{l \in L} F_{l}\left(\overline{y_{l}}\right)=f(\mu(T))$ with $\overline{y_{l_{m}}^{m}}>0$ to get that the partial derivatives of $f$ and $F_{l_{m}}$ with respect to the $m$-th coordinate satisfy $f^{m}(\mu(T))=F_{l_{m}}^{m}\left(\bar{y}_{l_{m}}\right)$.

The two implications yield $(\nabla f)(\mu(T)) \geq p^{*}$.

$f \in \mathscr{F}$, and hence $f$ is homogeneous of degree one, and so, by Euler $f(\mu(T))=$ $\nabla f(\mu(T)) \mu(T)$. Since also $f(\mu(T))=\sum_{l} F_{l}\left(\bar{y}_{l}\right)=p^{*} \sum_{l} \bar{y}_{l}=p^{*} \mu(T)$,

$$
\mu(T)\left[\nabla f(\mu(T))-p^{*}\right]=0
$$

As $\mu(T) \in \mathbb{R}_{++}^{M}$ and $p^{*} \leq \nabla f(\mu(T))$, it follows that $p^{*}=\nabla f(\mu(T))$.

Corollary 5 The core of the market game $\hat{V}$ with $F_{l} \in \mathscr{F} \forall$ l contains $x^{*}$, the t.u.c.e. payoff distribution.

Proof By Proposition 4, $u^{*}=x^{*}$; by Lemma $1, x^{*}$ is in the core of $V$. But $\hat{V} \leq$ $f \circ \mu=V$ and $\hat{V}(T)=V(T)$, so $x^{*}$ is in the core of $\hat{V}$. 


\section{Comments}

To restate: our main result is that if there is more than one big player and $r \geq 2$ in a smooth symmetric economy, the asymptotic nucleolus assigns to every small player the same allocation as his competitive payoff. One could interpret the result as reflecting the fierce competition among the big players (e.g., à la Bertrand), where the exploitation of the small players disappears in the core and, therefore, in the nucleolus. However, the Shapley value might assign a better allocation to a big player than his competitive payoff in contrast to the monopoly case. However, this is not true in general, as our examples show.

Note that the Shapley value in Proposition 2 for a simple duopoly corresponds to a heuristic based on the main result of Fogelman and Quinzii (1980), who show that the asymptotic Shapley value for scalar games of bounded variation (converging to a mixed game) is one of the values of the mixed game introduced by Hart (1973), and later fully characterised by Haimanko (2000) (as "path values"): ${ }^{3}$ imagine that the entrance position of every big player is uniformly and independently distributed on the unit interval and the small players join the coalition anywhere in-between. Indeed, then for the mixed game, the Shapley value allocates $\int_{0}^{1} \int_{t}^{1} h_{1}(t) d z d t+\int_{0}^{1} \int_{0}^{t} h_{2}(t) d z d t=$ $\int_{0}^{1}\left[(1-t) h_{1}(t)+t h_{2}(t)\right] d t$ to a big player. In order to find the asymptotic value one could potentially use a scalar representation of the game as in the proof of Proposition 6 , however we use a direct computation in Proposition2, which is sufficiently easy in our case.

The Shapley value to the syndicate (a single big) player in Guesnerie (1977) is also consistent with this heuristic calculation, and it can be obtained directly using a similar argument to the one in the proof of Proposition2, which can be extended to any number of big players of the same type.

\section{Appendix A: Regular production functions}

Proposition 5 For a collection $\left(F_{l}\right)_{l=1}^{L}$ of functions such that $F_{l} \in \mathscr{F} \forall l$, define $f: \mathbb{R}_{+}^{M} \rightarrow \mathbb{R}_{+}$by $z \mapsto \max _{\left(y_{l}\right)_{l}}\left\{\sum_{l=1}^{L} F_{l}\left(y_{l}\right): \sum_{l=1}^{L} y_{l} \leq z\right\}$. Then $f \in \mathscr{F}$ and $f^{m}(z)=F_{l}^{m}\left(y_{l}\right)$ for $m, l:\left(y_{l}\right)_{m}>0$, and for $\left(y_{l}\right)_{l}$ such that $f(z)=\sum_{l=1}^{L} F_{l}\left(y_{l}\right)$ and $\sum_{l=1}^{L} y_{l} \leq z$.

Proof Since the set $\left\{\left(y_{l}\right)_{l \in L} \in \mathbb{R}_{+}^{M L}: \sum_{l=1}^{L} y_{l} \leq z\right\}$ is compact and all $F_{l}$ are continuous, the maximum is attained. Concavity of $f$ follows from that of $F_{l}$ and

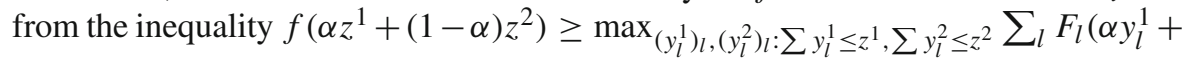
$\left.(1-\alpha) y_{l}^{2}\right)$ for $\alpha \in[0,1]$. Clearly, $f(0)=0$ and $f$ is non-negative and super-additive. Next, we show continuity of $f$ on $\mathbb{R}_{+}^{M}$.

Since $f$ is concave it is also continuous on $\mathbb{R}_{++}^{M}$. Pick a sequence $\left(z^{k}\right)_{k}$ converging to a point on the boundary, $z^{*} \in \mathbb{R}_{+}^{M}$. If $z^{*}=0$, the conclusion follows immediately

\footnotetext{
3 The general results about existence of asymptotic Shapley value are systematised in Neyman (2002).
} 
since $F_{l}(0)=0 \forall l$. Assume now $z^{*} \neq 0$. Let $\hat{z}^{k} \equiv z^{k} \wedge z^{*}$, i.e., $\hat{z}_{m}^{k} \equiv \min \left\{z_{m}^{k}, z_{m}^{*}\right\}$ for every $m \in\{1, \ldots, M\}$. So, $\hat{z}^{k} \underset{k \rightarrow \infty}{\rightarrow} z^{*} \wedge z^{*}=z^{*}$. Denote by $z_{+}^{*}$ the vector composed of strictly positive components of $z^{*}$, so $z_{+}^{*} \in \mathbb{R}_{++}^{n}$, where $n$ is the number of elements in the set $\left\{m: z_{m}^{*}>0\right\}$. Let $\hat{f}$ be the restriction of $f$ to $\mathbb{R}_{+}^{n}$, i.e., $\hat{f}(x)=f(x, 0)$, where $x \in \mathbb{R}_{+}^{n}$ and $(x, 0) \in \mathbb{R}_{+}^{M}$. Then $\hat{f}$ too is concave and therefore continuous on $\mathbb{R}_{++}^{n}$. Similarly, let $\hat{z}_{+}^{k}$ be the vector composed of strictly positive components of $\hat{z}^{k}$. Since $\hat{z}^{k} \rightarrow z^{*}$, for $k$ large enough, $\hat{z}_{+}^{k} \in \mathbb{R}_{++}^{n}$. But $f$ is non-decreasing, since $F_{l}$ are so by Remark 1 , hence $f\left(z^{k}\right) \geq \hat{f}\left(\hat{z}^{k}\right) \rightarrow \hat{f}\left(\hat{z}^{*}\right) \equiv f\left(z^{*}\right)$. We conclude that $\lim \inf f\left(z_{k}\right) \geq f\left(z^{*}\right)$.

To complete the proof of continuity consider again the converging sequence, $z^{k} \rightarrow$ $z^{*}$ and let $\left(y_{l}^{k}\right)_{l \in L}$ be such that $z_{k}=\sum_{l=1}^{L} y_{l}^{k}$ with $f\left(z^{k}\right)=\sum F_{l}\left(y_{l}^{k}\right)$. By construction, for every $l$ the sequence $y_{l}^{k}$ is bounded, hence is convergent, so $y_{l}^{k} \rightarrow y_{l}$, extracting a subsequence if necessary. Continuity of $F_{l}$ yields $f\left(z^{k}\right)=\sum F_{l}\left(y_{l}^{k}\right) \rightarrow \sum F_{l}\left(y_{l}\right)$, and since $\sum y_{l}=z^{*}, f\left(z^{*}\right) \geq \sum F_{l}\left(y_{l}\right)$, by construction of $f$. Thus we obtain $f\left(z^{*}\right) \geq \lim \sup f\left(z^{k}\right)$. Therefore $f$ is continuous.

It is left to prove existence of partial derivatives $f^{m}\left(z^{*}\right)$ with respect to strictly positive coordinates. Let $z^{*} \in \mathbb{R}_{+}^{M}$ with $z_{m}^{*}>0$. So we need to show that $f^{m}\left(z^{*}\right)$ exists.

First, note that by concavity of $f$ it is enough to show that its partial derivative from the left $\left(D_{m}^{-} f\right)\left(z^{*}\right)$ equals the partial derivative from the right $\left(D_{m}^{+} f\right)\left(z^{*}\right)$, or, using the definitions that $\lim _{\varepsilon \rightarrow 0^{+}} \frac{f\left(z^{*}+\varepsilon e_{m}\right)-f\left(z^{*}\right)}{\varepsilon}=\lim _{\varepsilon \rightarrow 0^{+}} \frac{f\left(z^{*}\right)-f\left(z^{*}-\varepsilon e_{m}\right)}{\varepsilon}$, where $e_{m}$ is the $m$-th unit vector.

Let $\left(y_{l}\right)_{l \in L}$ be such that $f\left(z^{*}\right)=\sum F_{l}\left(y_{l}\right)$ where $\sum y_{l} \leq z^{*}$. Take $m, l^{*}$ such that $\left(y_{l^{*}}\right)_{m}>0$, and hence $z_{m}^{*}>0$. Obviously $z^{*}+\varepsilon e_{m}=\sum_{l^{\prime} \neq l^{*}} y_{l^{\prime}}+\left(y_{l^{*}}+\varepsilon e_{m}\right)$ and $z^{*}-\varepsilon e_{m}=\sum_{l^{\prime} \neq l^{*}} y_{l^{\prime}}+\left(y_{l^{*}}-\varepsilon e_{m}\right)$. Therefore, by definition of $f$ we have: $f\left(z^{*}+\varepsilon e_{m}\right) \geq \sum_{l^{\prime} \neq l^{*}} F_{l^{\prime}}\left(y_{l^{\prime}}\right)+F_{l^{*}}\left(y_{l^{*}}+\varepsilon e_{m}\right)$ and $f\left(z^{*}-\varepsilon e_{m}\right) \geq \sum_{l^{\prime} \neq l^{*}} F_{l^{\prime}}\left(y_{l^{\prime}}\right)+$ $F_{l^{*}}\left(y_{l^{*}}-\varepsilon e_{m}\right)$. This implies

$$
\begin{aligned}
\lim _{\varepsilon \rightarrow 0^{+}} \frac{f\left(z^{*}+\varepsilon e_{m}\right)-f\left(z^{*}\right)}{\varepsilon} & \geq \lim _{\varepsilon \rightarrow 0^{+}} \frac{F_{l^{*}}\left(y_{l^{*}}+\varepsilon e_{m}\right)-F_{l^{*}}\left(y_{l^{*}}\right)}{\varepsilon}=F_{l^{*}}^{m}\left(y_{l^{*}}\right) \\
\lim _{\varepsilon \rightarrow 0^{+}} \frac{f\left(z^{*}\right)-f\left(z^{*}-\varepsilon e_{m}\right)}{\varepsilon} & \leq \lim _{\varepsilon \rightarrow 0^{+}} \frac{F_{l^{*}}\left(y_{l^{*}}\right)-F_{l^{*}}\left(y_{l^{*}}-\varepsilon e_{m}\right)}{\varepsilon}=F_{l^{*}}^{m}\left(y_{l^{*}}\right)
\end{aligned}
$$

Thus, $\left(D_{m}^{+} f\right)\left(z^{*}\right) \geq\left(D_{m}^{-} f\right)\left(z^{*}\right)$. By the concavity of $f$ we have $\left(D_{m}^{+} f\right)\left(z^{*}\right) \leq$ $\left(D_{m}^{-} f\right)\left(z^{*}\right)$. Therefore $f^{m}\left(z^{*}\right)=F_{l^{*}}^{m}\left(y_{l^{*}}\right)$.

It follows that $f \in \mathscr{F}$.

\section{Appendix B: Existence of Shapley value for a simple duopoly game}

Proposition 6 Let $f: \mathbb{R}_{+}^{M} \rightarrow \mathbb{R}$ be concave, non-decreasing with $f(0)=0$ and continuous at zero. Let $T_{0}=[0,1], T_{1}=\left\{a_{1}, a_{2}\right\}, \lambda_{0}$ be the Lebesgue measure on 
$[0,1], \lambda_{1}\left(a_{i}\right)=1$. For any $S \in \Sigma$, let $\mu(S)=\lambda_{0}([0,1] \cap S) \omega_{0}+\lambda_{1}\left(\left\{a_{1}, a_{2}\right\} \cap S\right) \omega_{1}$ such that $\omega_{0}+2 \omega_{1} \in \mathbb{R}_{++}^{M}$.

Then the game $V=f \circ \mu$ has an asymptotic Shapley value.

\section{Proof}

$$
\text { Let }^{4} g(x)= \begin{cases}f\left(x \omega_{0}\right) & x \in I_{1}=[0,1] \\ 0 & x \in I_{2}=(1,2) \\ f\left((x-2) \omega_{0}+\omega_{1}\right) & x \in I_{3}=[2,3] \\ 0 & x \in I_{4}=(3,4) \\ f\left((x-4) \omega_{0}+2 \omega_{1}\right) & x \in I_{5}=[4,5]\end{cases}
$$

Let $\sigma \stackrel{\text { def }}{=} \lambda_{0}+2 \lambda_{1}$. Note, that the difference between $\sigma(S)$ and its integer part is $\lambda_{0}$, while the integer part equals $2 \lambda_{1}$, whenever $\lambda_{0}<1$. When $\lambda_{0}=1$ then $\sigma(S) \in\{1,3,5\}$ thus identifying the value of $\lambda_{1} \in\{0,1,2\}$. Hence, the range of $\sigma$ is $[0,1] \cup[2,3] \cup[4,5]$ and every element of the range uniquely identifies the pair $\lambda_{0}, \lambda_{1}$. It follows then that $g \circ \sigma$ is well-defined, and $f \circ \mu=g \circ \sigma$ on $\Sigma$. Since, by continuity of $f$ at zero and construction of $g, \lim _{x \rightarrow 0} g(x)=\lim _{x \rightarrow 0} f\left(x \omega_{0}\right)=f(0)=g(0)=0$ and by concavity of $f$ (hence its continuity in the interior), $\lim _{x \rightarrow 5} g(x)=\lim _{x \rightarrow 5}$ $f\left((x-4) \omega_{0}+2 \omega_{1}\right)=f\left(\omega_{0}+2 \omega_{1}\right)=g(5), g$ is continuous at the corners and is zero at zero. $g$ is of bounded variation: indeed, by monotonicity of $f, g$ is monotonic on each of the intervals $I_{1}, I_{3}, I_{5}$, respectively, while being zero elsewhere, so the total variation of $g$ is $2(f(1)+f(3))+f(5)<\infty$.

Following Hart (1973), define $b v^{\prime}$ as a set of real-valued functions of bounded variation defined on $[0,1]$ that are zero at zero and are continuous at 0 and 1 , so, when normalised, $g \in b v^{\prime}$. Since also $\sigma \in F L$, with $F L$ the space of measures that can be represented as a sum of non-atomic measure and a measure with a finite carrier, $g \circ \sigma \in b v^{\prime} F L$. But since by Neyman (1979) (cf. Neyman 2002, thm. 4) $b v^{\prime} F L \subset A S Y M P$, the conclusion follows.

\section{References}

Aumann R (1964) Markets with a continuum of traders. Econometrica 32:39-50

Aumann R, Shapley L (1974) Values of non-atomic games. Princeton University Press, Princeton, NJ

Einy E, Moreno D, Shitovitz B (1999) The asymptotic nucleolus of large monopolistic market games. J Econ Theory 89(2):186-206

Fogelman F, Quinzii M (1980) Asymptotic value of mixed games. Math Oper Res 86-93

Gabszewicz J, Shitovitz B (1992) The core in imperfectly competitive economies. In: Aumann RJ, Hart S (eds) Handbook of game theory with economic applications, vol 1. Elsevier, North-Holland, pp 459-483

Guesnerie R (1977) Monopoly, syndicate, and shapley value: about some conjectures. J Econ Theory $15(2): 235-251$

Haimanko O (2000) Partially symmetric values. Math Oper Res 25(4):573-590

Hart S (1973) Values of mixed games. Int J Game Theory 2(1):69-85

Mas-Collel A (1985) The theory of general economic equilibrium: a differentiable approach. Cambridge University Press, Cambridge

Neyman A (1979) Asymptotic values of mixed games. In: Moeschlin O, Pallaschke D (eds) Game theory and related topics. North-Holland, pp 71-81

Neyman A (2002) Values of games with infinitely many players. In: Aumann R, Hart S (eds) Handbook of game theory with economic applications, vol 3. Elsevier, North-Holland, pp 2121-2167

\footnotetext{
4 We wish to thank the anonymous referee for an idea leading to this construction.
} 
Market games with large players

Neyman A, Smorodinsky R (2004) Asymptotic values of vector measure games. Math Oper Res 29(4):739_ 775

Schmeidler D (1969) The nucleolus of a characteristic function game. SIAM J Appl Math 17(6):1163-1170

Shapley LS, Shubik M (1969) On market games. J Econ Theory 1(1):9-25

Shitovitz B (1973) Oligopoly in markets with a continuum of traders. Econometrica 41(3):467-501

Trockel W (1976) A limit theorem on the core. J Math Econ 3(3):247-264 